\title{
Benign Retropharyngeal Neoplasm
}

National Cancer Institute

\section{Source}

National Cancer Institute. Benign Retropharyngeal Neoplasm. NCI Thesaurus. Code C162826.

A benign neoplasm that arises from the retropharyngeal space. 